\title{
Imagery Beauty in the Application of Microscopic Material Forms
}

\author{
Du Yiming \\ South China University of Technology, Institute of Micro-Science Arts, Guangzhou, China, C2170010
}

Keywords: Microscopic material form; imagery; imagery beauty

\begin{abstract}
With the diversified development of art forms, the combination of art and science shows a booming trend. Applying the microscopic material form to the creation of paintings is beyond the boundaries of vision. It is a comprehensive experience of sensory and a new expression of the combination of subjective emotions and objective objects. This way of creation is both improvised and well designed. This paper reflects the cultural connotation of the application of microscopic material form by analyzing the microscopic material form and image beauty.
\end{abstract}

At present, the two disciplines of science and art are developing more and more closely. Scientific art and artistic science have become an irresistible trend. Through the observation of scientific instruments, such as microscopes, the microscopic world has become a new field in which people continue to expand their visual boundaries. Microscopic science is not only a science and technology subject,it has opened up a new world in the field of art. This field of vision has an unexpected visual effect. It presents an image beauty which is completely different from the macro world. Its texture and structural beauty also open a new door for artistic creation, providing unlimited possibilities. These microscopic forms give people a visual and spiritual shock with their unique charm, which allows people to rethink macro and micro. Artistic creation no longer only seeks material from the macroscopic world which is visible to the naked eye, but instead looks at the strange microscopic material world.

Applying the microscopic material form to the creation and design of painting, as an abstract artistic expression, this language is more like a new expression of the combination of subjective emotions and objective objects. The point, line, and surface of the shape are reflected in a new structure, rather than a complete replica. This kind of creation has gone beyond the boundaries of vision and has become a comprehensive experience of perception, blending into the design of images and textures. It includes improvisation and well-designed art, combines art and science to express the inner world of the creator. Therefore, the beauty of imagery in the application of microscopic material forms has become an indispensable link in the study of microscopic art.

\section{Introduction}

\subsection{Imagery}

The influence of a long history and culture has produced a unique aesthetic taste of Chinese art. The fusion of objective objects and subjective emotions forms a unique artistic language: imagery. As a kind of aesthetics with rich connotations in China, it appears in all fields of art. The origin of the imagery concept can be traced back to the "Yi Zhuan": "The saints set up image to express idea, set up divinatory symbols to express the truth, and make words to express their opinion." It means: saints create divinatory symbols to express the meaning of God, to show the truth, to express viewpoint. The "imagery" refers to the will of the Holy Body, and "image" refers to the symbolic phenomenon. Although it is far from the aesthetic category, it uses "image" as a symbolic system that can express opinions for the descendant. And the formation of the "image" aesthetic concept has laid a certain foundation of later research.

The first time to combine "meaning" and "image" was the "Lun Heng·Luan Long Pian" in the Han Dynasty: "On the canvas paints the image of bear, elk, tiger, leopard and so on. The cloth is named Hou, the meaning of the rite is precious. The name depends on the meanings." It is to say: 
draw pictures of bear, elk, tiger, leopard and other beasts on the canvas, let the emperor shoot the bear, the princes shoot the elk, the ministers shoot the tiger and leopards, the gentlemen go to shoot the deer and the pigs. The beasts they can shoot shows their bravery. The canva with the image of the bear on it is called Hou, it means overthrow the fatuity. The name of the canvas is according to the ritual system, and the ritual system is conveyed from the images on the canvas. But the word "image" here refers to the animal pattern painted on the ancient archery target, it stands for the symbolic image.

The concept of "imagery" was first proposed in Liu Wei's "Wen Xin Diao Long· Shen Si Pian" in the Southern and Northern Dynasties, and it also established the aesthetic category. In the article it says: "The master of solo is watching the image and carrying the weight at the glimpse of the object." It means that a uniquely crafted craftsman can swing an axe in the image of the heart. The "image" here refers to the image formed by the thoughts, which means that the image of the object must be integrated with the creator's own interest and emotion. In the art creation after this, the concept of imagery was widely used to form a unique aesthetic consciousness of Chinese art. "Imagination" has also become an important criterion for evaluating art works, and has become a basic aesthetic category.

\subsection{Imagery beauty}

The so-called "meaning" is the inner thought, and the so-called "image" is the external representation and expression. The image is to express the spiritual pursuit that exists at the ideological level with concrete manifestations. This form of creative expression is not to reproduce the image of reality as seen on the painting, nor to subconsciously smear beyond the realistic image of the real thing, but to establish a spiritual image from abstract thinking and select appropriate representations. The image comes with a smile. The picture itself may be subtle, but the author's thoughts have already reached the realm of the image in the painting. In the art work that truly stands for art, the beauty of imagery is often reflected in people's own perceptions. This kind of sentiment is a collision between the experience of nature and the course of one's own thoughts. The spark of the excitement is instantaneous, and retaining this great impulse by means of the image will be expressed on paper, giving the viewer a shock. The space and the shock of the visual, this is the beauty conveyed through the image. "A thousand readers have a thousand Hamlet." Each viewer has different feelings in the face of the same piece of work because of his own cultural knowledge and life background. The beauty of imagery is an experience of "only can be understood, cannot be said" under the guidance of thinking, and is the subjective feeling of the aesthetic subject to the aesthetic object. The soul collision of the spiritual world caused by these visual feelings, is it not the beauty of the imagery of paintings?

\subsection{Microscopic material form}

In the process of scientific development and progress, the emergence of the micro world has provided artists with new situations and modes of thinking. Through the micro-science, the door of the micro-art world has been opened. The micro-forms presented have their original intentions, and they can all be given the beauty of images through the display of micro-forms. Expanded the source of painting materials for artists. For everyday materials captured by the naked eye or captured by ordinary imaging, micro-scientific research has seen a broader and deeper world that is less familiar. The huge microscopic material world, the imagination and creative inspiration brought about by artistic creation cannot be replaced by other feelings. Applying the forms discovered by micro-science to art creation and design, promoting interdisciplinary research, enriching the theoretical knowledge combined with micro-science and artistic creation helps to construct a comprehensive and systematic theoretical system, inspiring viewers from art works. It is of great significance to think about the nature of life and life, and to scientifically and scientifically. The application of microscopic material forms makes the composition of the picture clever, the color, space and shape are very mysterious, and the small part is magnified in the grand natural environment, giving the natural small objects a grand sense of the universe, implanting the spirit meaning, through the passenger Viewing objects express cultural ideas and artistic spirit. The use of 
microscopic forms for artistic creation not only shows the combination of science and technology and artistic creation, but also a new way and method in the field of painting creation. The works created by it will inevitably enrich the diversity and contemporaneity of today's painting art, create new and far-reaching status and exert huge influence.

\section{The embodiment of image beauty in the application of microscopic material form}

\subsection{Possibility of microscopic material forms’imagery}

Ye Lang said in "The Beauty in Imagery - The Basic Principles of Aesthetics": "The ontology of art is an aesthetic image that is a complete and meaningful sensible world. Art does not provide people with a valuable instrument, nor does it use a form of proposition to provide people with a truth about the world, but to present an image world to people, so that the audience can have a sense of beauty." He believes that art and beauty are inseparable, and the difference between art and non-art depends on if the work can present an image world. The microscopic material form can trigger people to perceive the rhythm of life, experience the tension of natural life, explore the traces of the thrilling life activities, and trigger the philosophical thinking of the meaning of life in the depths of people's minds.

The tiny structures, textures, tissues, and so on in the microscopic material form give people a mysterious senses and the possibility of microscopic material forms 'imagery, because of its unique existence form and structural outline. Such as:

Figure 1 is the cross section of the human blood vessels under the microscope, it presents a beautiful color and a mysterious arrangement. The viewer sees a wonderfully structured world that has nothing to do with human blood vessels. Beyond human perception, abstract and complex, with a natural and vivid structure. Exaggerated and bright colors, the contrast is strong but presents a unique harmony, which causes the viewer to think about life. It is also like the vast interstellar, mysterious and profound, giving people the enlightenment of the origin of life. Such psychological activities have inspired the artist's creative inspiration and laid the foundation for the search for creative content and artistic expression.

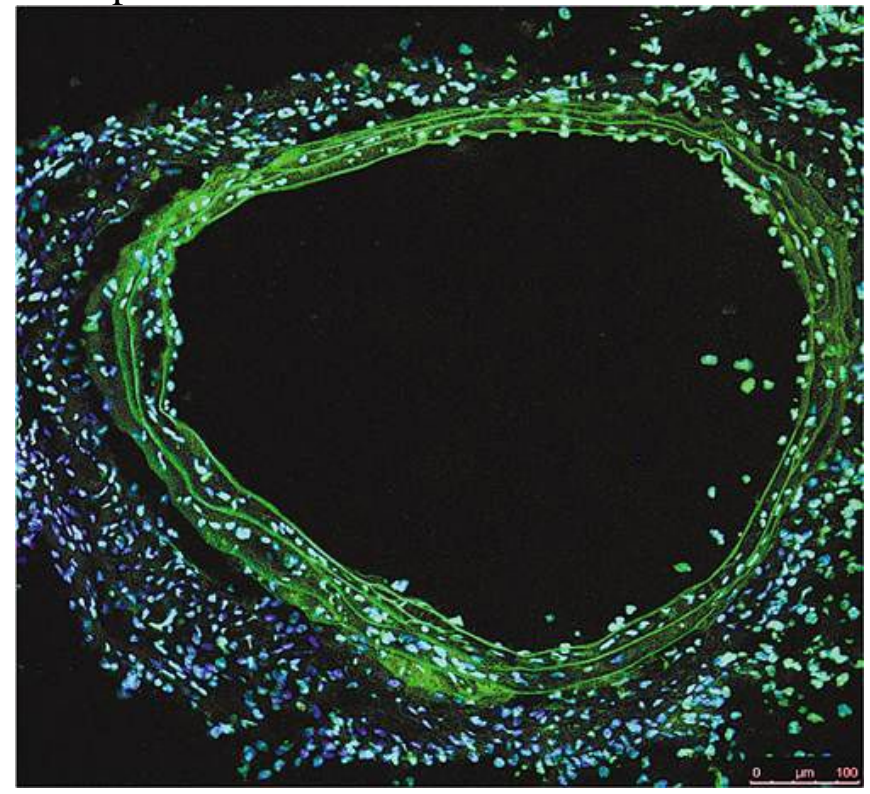

Figure 1 Human blood vessel cross section under the microscope

Figure 2 is the tiny crystals of oxycodone (narcotic drugs), it seems like the shape of flying petals and dancing bees under the microscope. The colors are harmonious and unified, the structure is exquisite and rich, and it is natural and vivid. The structure and color of the microscopic material form presents a light and lively life rhythm, which provides reference for artistic creation and reference for imagery beauty. 


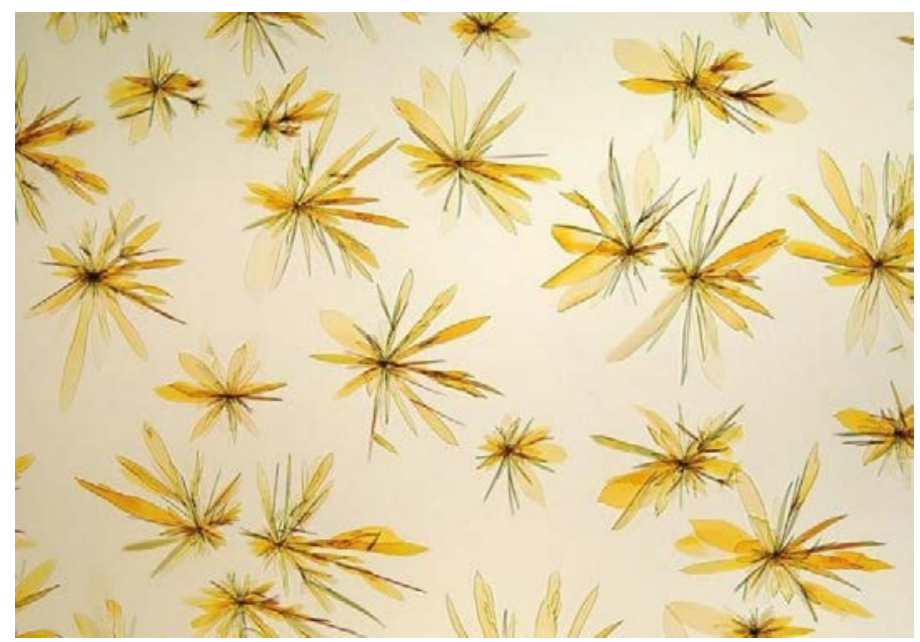

Figure 2 Oxycodone (narcotic drugs) tiny crystals

Figure 3 is the crystal structure of the copper under the microscope, the exterior shape is clear, and the sharp facet is sharply turned. Applying this microscopic material form to artistic creation, it expresses the sharp and hard texture of metal products, emphasizing the perseverance personality just like the bronze sword.

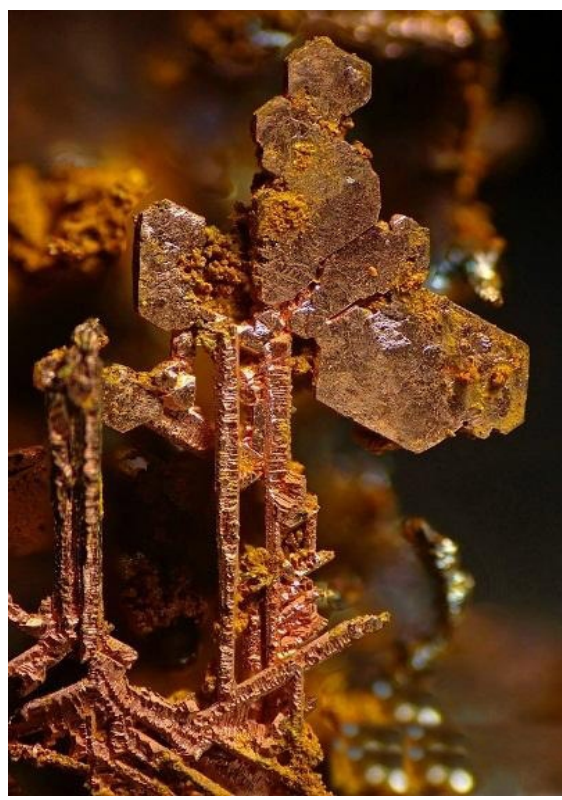

Figure 3 Copper crystal structure under the microscope

The numerous microscopic material forms, each of which can be explored for its unique aesthetic styling and emotional orientation. The composition of similar geometric elements awakens our desire to seek knowledge from the microscopic material world. The microscopic material, the external form is rich and varied, and the inner also carries the mysterious visual impact. They trigger the viewer's philosophical thinking on the nature of life, the perception and resonance of the rhythm of life, manifests the possibility of microscopic material forms' imagery.

\subsection{Microscopic material form and image beauty}

Hegel put forward the famous proposition in his "Aesthetics": "Beauty is the perceptual manifestation of the concept." He believes that beauty is the idea, beauty must be true, and it is a perceptual expression of truth. The idea itself exists only as a universal truth. It must be expressed in the concrete form of the sensible thing, and it becomes a work of art that can be appreciated by people. Zhu Guangqian, a famous contemporary esthetician, said: "The world of beauty is purely an image world." Zong Baihua, an esthetician, once said: "The subjective life sentiment blends with the objective natural scene, making a flying, leaping, lively spiritual environment." This emotional 
point that has been repeatedly scrutinized by estheticians is the beauty of artistic imagery. As Ye Lang said in the article "America in the Image", this kind of beauty "is not a physical reality, nor an abstract conceptual world, but a complete, full of meaning and full of funny world."

The application of microscopic material morphology under microscopic scientific research is to use modern science and technology to extract the color, shape, structure and texture of microscopic materials, and apply them in the artistic creation. This process is the author's sensibility creation combined with his own thoughts and understanding. In this process, the "truth" of microscopic material is conveyed to the viewer through the "sensible" understanding of the creator, forming a series of beautiful artistic labor.

\section{Conclusion}

Micro-science art is using the high-tech means to discover the mysterious and bizarre microcosm which is beyond the naked eye, reveal the microscopic natural form, and extract the aesthetic elements of microscopic form, and create unique microscopic artistic creation. This interdisciplinary experimental creation poetically explains the spiritual beauty of the objective material world. The imagery beauty of microscopic material form is the transcendence of the real thing, the sublimation of the figurative thing, it is the new soul of the art work. The works are not spoken, but the aesthetic image makes the works reflect the strong spiritual power from the concrete objects.

\section{Acknowledgement}

This research is based on the Study of Micro-Science Arts Form (Serial number: C2170010).

\section{References}

[1] Zhang Shiying. The Philosophical Ontological Foundation of the Beauty of Imagery [J]. Chinese Literature Review, 2017(09):8-13.

[2] Ye Lang. Beauty in Imagery-Abstract of Basic Principles of Aesthetics [J]. Journal of Peking University (Philosophy and Social Sciences), 2009, 46(03):11-19.

[3] Yu Zhihong. Microscopic Natural Forms of Imagery [J]. Journal of Nanjing University of the Arts (Art and Design Edition), 2009(06):103-105.

[4] Shang Xingjie. Life is beautiful but not words--Analysis of decorative language in microscopic imagery of life [J]. Art and Design (Theory), 2014, 2(08):118-119.

[5] Hu Jing. The Image Creation of Chinese Painting Art [J]. Art Education, 2006 (01): 91. 